\title{
Arco aórtico derecho, divertículo de Kommerell y arteria subclavia izquierda aberrante
}

\author{
Right aortic arch, Kommerell's diverticulum \\ and aberrant left subclavian artery
}

\author{
I. Simón-Yarza, G. Viteri-Ramírez, J. Etxano, P. Slon, M. Ferreira, G. Bastarrika
}

\section{RESUMEN}

La arteria subclavia derecha aberrante o "arteria lusoria" es la variante anatómica más común del desarrollo embrionario de la aorta y sus ramas, llegando a estar presente en un 0,5-2\% de la población; con menos frecuencia se puede encontrar una arteria subclavia izquierda aberrante asociada al arco aórtico derecho. Estas variantes anatómicas deben incluirse en el diagnóstico diferencial del ensanchamiento mediastínico superior visible en la radiografía de tórax. Se presenta el caso clínico de un paciente que presentaba arco aórtico derecho y arteria subclavia izquierda aberrante con dilatación en su origen (divertículo de Kommerell) como causa de ensanchamiento mediastínico superior de etiología incierta detectado de manera incidental en una radiografía de tórax realizada en un paciente con sintomatología respiratoria.

Palabras clave. Arteria subclavia izquierda aberrante. Divertículo de Kommerell. Arco aórtico derecho.

\begin{abstract}
The right aberrant subclavian artery or "arteria lusoria" is the most common anatomical variant of the embryonic development of the aorta and its branches, with a presence in $0.5-2 \%$ of the population. Less frequently, a right aortic arch with aberrant left subclavian artery may be present. These anatomical variations should be included in the differential diagnosis of superior mediastinal widening seen on chest radiographs. In this report, we present a right aortic arch with left aberrant subclavian artery dilated at its origin (Kommerell's diverticulum) as a cause of superior mediastinal widening detected incidentally on a chest radiograph.
\end{abstract}

Key words. Aberrant left subclavian artery. Kommerell's diverticulum. Right aortic arch.
Servicio de Radiología. Clínica Universidad de Navarra.

Recepción: 2 de diciembre de 2010

Aceptación provisional: 14 de febrero de 2011

Aceptación definitiva: 28 de febrero de 2011

\section{Correspondencia}

Isabel Simón Yarza

Clínica Universidad de Navarra

Servicio de Radiología

Avda. Pío XII, 36

31008 Pamplona

E-mail.msyarza@unav.es 


\section{INTRODUCCIÓN}

Los arcos aórticos que acompañan a los arcos faríngeos sufren sucesivas modificaciones durante el desarrollo embrionario hasta alcanzar la anatomía definitiva de la aorta y sus ramas ${ }^{1}$. Variaciones en la regresión de los arcos aórticos dan lugar a diferentes anomalías del cayado aórtico (Tabla 1) ${ }^{2}$. Entre estas anomalías se encuentra el cayado aórtico derecho y la arteria subclavia aberrante (ASA). Habitualmente, la ASA tiene un recorrido retroesofágico que puede condicionar la aparición de disfagia por compresión. Presentamos el caso de un paciente con disnea que mostraba arco aórtico derecho con arteria subclavia izquierda aberrante que producía compresión del esófago y la tráquea.

Tabla 1. Clasificación de las anomalías congénitas del arco aórtico y de las variantes anatómicas de los troncos supraórticos

\begin{tabular}{l}
\hline Anomalías congénitas del arco aórtico \\
\hline Arco aórtico izquierdo con arteria subclavia derecha aberrante \\
Arco aórtico derecho con ramificación en espejo \\
Arco aórtico derecho con arteria subclavia izquierda aberrante \\
Arco aórtico cervical \\
Arco aórtico interrumpido \\
\hline Variantes anatómicas de los troncos supraórticos \\
\hline Origen común del tronco braquiocefálico arterial y la arteria carótica común izquierda \\
Origen de la arteria carótida común izquierda en el tronco braquiocefálico arterial \\
Arteria vertebral izquierda aberrante (origen en el cayado aórtico)
\end{tabular}

\section{CASO CLÍNICO}

Varón de 76 años, diabético, hipertenso, exfumador desde hace 7 años de 80 paquetes-año. Acudió a nuestro centro por un cuadro de tos seca y disnea de moderados esfuerzos de dos meses de evolución que no había experimentado mejoría con tratamiento antibiótico. Se le realizó una radiografía de tórax en la que se objetivó un ensanchamiento mediastínico superior derecho con importante desplazamiento de la tráquea. Dados los factores de riesgo del paciente, su sintomatología clínica y el hallazgo descrito en la radiografía de tórax se decidió completar el estudio con una tomografía computarizada (TC). La TC con contraste intravenoso yodado demostró una anomalía anatómica del cayado aórtico y de los troncos supraórticos como causa del ensanchamiento mediastínico superior. El paciente presentaba un arco derecho con un origen aberrante de la arteria subclavia izquierda a partir de un divertículo de Kommerell. La arteria subclavia izquierda se encontraba dilatada en su origen y transcurría por detrás del esófago comprimiendo parcialmente el mismo y desplazando anteriormente la tráquea (Fig. 1). 


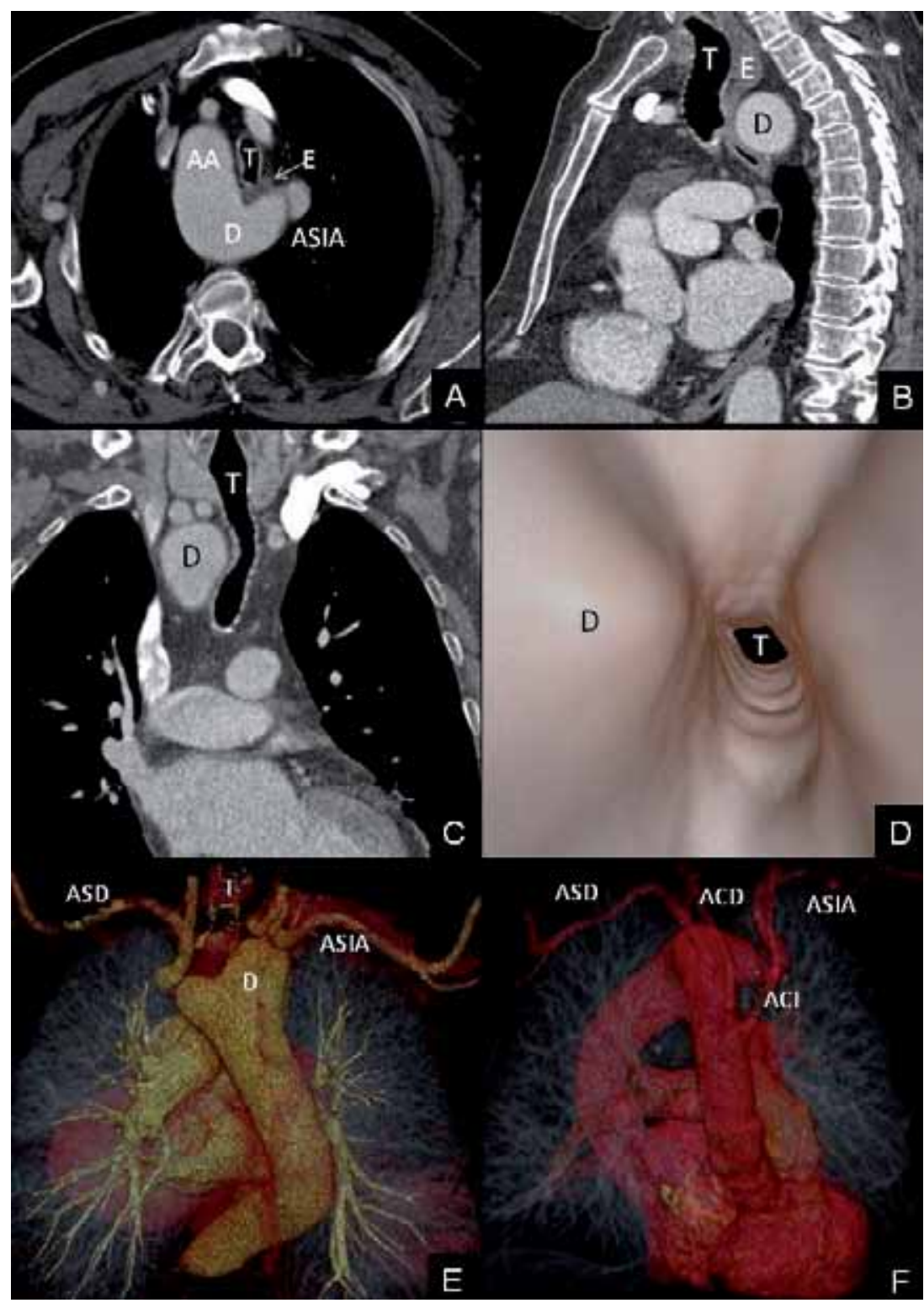

Figura 1. Estudio de TC tras la administración de contraste intravenoso. A. Plano axial oblicuo donde se observa el arco aórtico (AA) en el lado derecho y un divertículo de Kommerell (D) en el origen de la arteria subclavia izquierda aberrante (ASIA). El divertículo desplaza anteriormente la tráquea (T) y el esófago (flecha, E). B. Reconstrucción sagital en la que se observa el recorrido retroesofágico del divertículo de Kommerell (D). Nótese la compresión del esófago (E) entre el divertículo (D) y la tráquea (T). C. Reconstrucción coronal que muestra el desplazamiento lateral de la tráquea (T) provocado por el divertículo de Kommerell (D). D. Imagen de broncoscopia virtual en la que se observa la impronta y estrechamiento de la luz traqueal (T) causados por el divertículo de Kommerell (D). E, F. Reconstrucciones volumétricas. E. Visión posterior con arco aórtico derecho del que nacen la arteria subclavia derecha (ASD) y la arteria subclavia izquierda (ASIA) a partir del divertículo de Kommerell (D). F. Visión anterior del arco aórtico derecho del que se observa la salida consecutiva de las cuatro ramas supraórticas: arteria subclavia derecha (ASD), carótida derecha (ACD), carótida izquierda (ACI) y arteria subclavia izquierda aberrante (ASIA). 


\section{DISCUSIÓN}

Durante el desarrollo embrionario se forman seis pares de arcos aórticos simétricos que acompañan a los arcos faríngeos y desembocan en las aortas dorsales derecha e izquierda. En la región de los arcos aórticos las aortas son pares, fusionándose caudalmente en una única aorta descendente (Fig. 2a). A lo largo del desarrollo embrionario los arcos pierden su simetría tras sucesivas involuciones y variaciones en su recorrido. El arco aórtico final es el resultado de la interrupción del segmento dorsal del arco derecho que va desde la arteria subclavia derecha hasta la aorta descendente (Fig. 2b). Como norma general, en el lado derecho el cuarto arco y la séptima arteria intersegmentaria dan lugar a la arteria subclavia derecha, de la que se origina la carótida primitiva derecha (tercer arco); en el lado izquierdo, el cuarto arco da lugar al cayado aórtico del que nacen la carótida primitiva izquierda (tercer arco) y la arteria subclavia izquierda (séptima arteria intersegmentaria) (Fig. 2b) ${ }^{1}$. Variaciones en la localización de la interrupción y regresión de los arcos aórticos dan lugar a las diferentes anomalías congénitas del cayado aórtico (Tabla 1) como son el origen anómalo de la arteria subclavia derecha, el arco aórtico derecho, la arteria subclavia izquierda aberrante con arco aórtico derecho, el arco aórtico derecho con salida aislada de la arteria subclavia izquierda, la duplicación del cayado de la aorta, el arco aórtico cervical o el cayado aórtico interrumpido ${ }^{1,2}$.

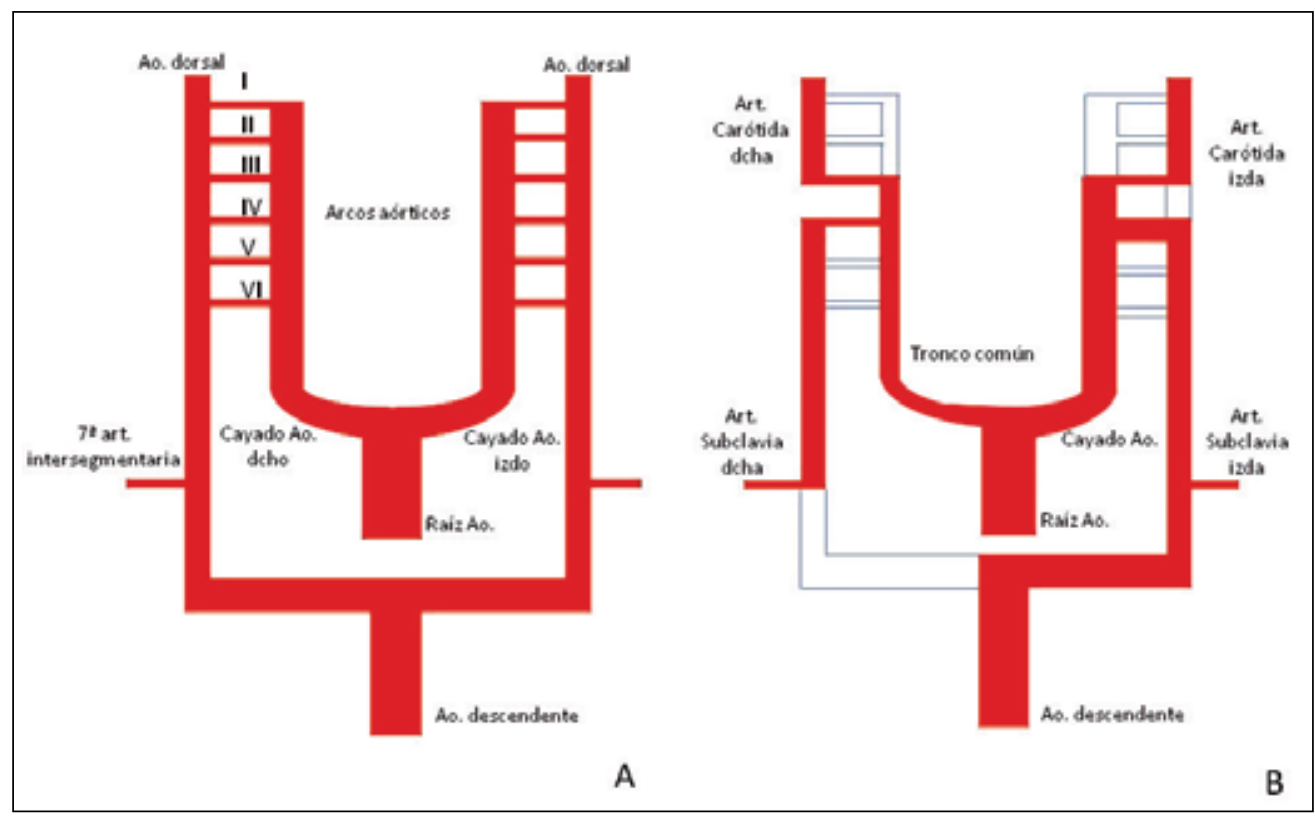

Figura 2. Esquema del sistema de doble arco aórtico durante el desarrollo embrionario. A. De la raíz aórtica nacen dos cayados aórticos simétricos que se comunican con las arterias dorsales a través de seis pares de arcos aórticos. Las aortas dorsales se fusionan caudalmente en una única aorta descendente. B. Esquema de la disposición de la aorta y los troncos supraórticos al final del desarrollo embrionario.

De entre todas estas anomalías congénitas el arco aórtico izquierdo con arteria subclavia derecha aberrante (ASDA), tam- bién conocida como "arteria lusoria", es la más frecuente, encontrándose presente en un $0,5-2 \%$ de la población. Esta anomalía es 
el resultado de la obliteración del cuarto arco aórtico entre la arteria carótida común derecha y la $\mathrm{ASDA}^{1,3,4}$. En esta variante anatómica nacen del cayado aórtico, consecutivamente, las arterias carótida derecha, carótida izquierda, subclavia izquierda y subclavia derecha. Ésta última atraviesa la línea media con un trayecto retroesofágico en el 85\% de los casos, lo que en ocasiones produce la llamada "disfagia lusoria» al quedar comprimido el esófago entre la tráquea y la arteria subclavia aberrante.

Por causas no bien conocidas, cuando el cayado aórtico se encuentra a la derecha $(0,1 \%$ de la población), es más común encontrar una "arteria lusoria" localizada en el lado izquierdo (arteria subclavia izquierda aberrante-ASIA) ${ }^{5}$, como en el caso que se presenta. Se trata de una variante que es imagen en espejo del cayado aórtico izquierdo con ASDA y, por tanto, puede producir la misma sintomatología. En estos pacientes, además, son más prevalentes las anomalías cardiacas estructurales, principalmente la tetralogía de Fallot ${ }^{6,7}$.

Aproximadamente dos tercios de los pacientes con ASDA o ASIA presentan una dilatación en su salida conocida como "divertículo de Kommerell", signo descrito por primera vez en el año 1936 en el contexto clínico de un paciente con disfagia ${ }^{8}$. El divertículo de Kommerell es un remanente del arco aórtico distal derecho del embrión y da lugar a una dilatación aneurismática en un $3-4 \%$ de los casos $^{3}$. Este divertículo puede ser sintomático por comprimir el esófago y producir disfagia lusoria o, lo que entraña mayor gravedad, puede provocar síntomas debido a la propia rotura del aneurisma; también se ha descrito compromiso respiratorio por compresión de la tráquea o del bronquio principal ${ }^{3,9}$ principalmente durante la edad pediátrica, cuando el árbol bronquial es menos resistente.

La gran mayoría de los pacientes con arteria subclavia aberrante se encuentran asintomáticos, siendo el diagnóstico un hallazgo casual al realizar una prueba de imagen por otros motivos. En caso de disfagia, su manejo clínico depende de la severidad de los síntomas. En situaciones de disfagia leve o moderada un tratamiento conservador modificando la dieta y administrando fármacos procinéticos puede ser suficiente ${ }^{10}$. El tratamiento quirúrgico está reservado para aquellos casos en que la disfagia sea severa o el riesgo de rotura del aneurisma sea elevado y consiste, habitualmente, en la resección de la arteria subclavia aberrante mediante esternotomía media o toracotomía y su anastomosis a la arteria carótida común ipsilateral ${ }^{11}$.

Como se muestra en el caso clínico que se presenta, el ensanchamiento mediastínico superior en una radiografía de tórax es un signo muy inespecífico. Éste puede deberse a múltiples causas como tumores mediastínicos, aneurismas o elongaciones vasculares, anomalías esofágicas o bocio. Por ello debe ser valorado adecuadamente en el contexto clínico de cada paciente. En el caso que se presenta la TC excluyó un tumor como causa del ensanchamiento mediastínico y permitió establecer el diagnóstico de arco aórtico derecho con arteria subclavia izquierda aberrante asociada a un divertículo de Kommerell. El conocimiento de esta variante anatómica resulta de gran interés a la hora de interpretar y orientar el diagnóstico de las posibles causas de un ensanchamiento mediastínico superior detectado de manera incidental en las radiografías de tórax tanto en personas asintomáticas como en pacientes que acuden al médico por disfagia.

\section{BIBLIOGRAFÍA}

1. SADLER TW. Langman. Embriología médica con orientación clínica. $8^{\underline{a}}$ ed. España: Madrid 2002.

2. Turkvatan A, Buyukbayraktar FG, Olcer T, CumHur T. Congenital anomalies of the aortic arch: evaluation with the use of multidetector computed tomography. Korean J Radiol 2009; 10: 176-184.

3. FreEd K, Low VH. The aberrant subclavian artery. Am J Roentgenol 1997; 168: 481-484.

4. Kleinman PK, SpevaK MR, Nimkin K. Left-sided esophageal indentation in right aortic arch with aberrant left subclavian artery. Radiology 1994; 191: 565-567. 
5. SHuford WH, Sybers RG, Edwards FK. The three types of right aortic arch. Am J Roentgenol Radium Ther Nucl Med 1970; 109: 67-74.

6. LuETMER PH, Miller GM. Right aortic arch with isolation of the left subclavian artery: case report and review of the literature. Mayo Clin Proc 1990; 65: 407-413.

7. Ramaswamy P, Lytrivi ID, Thanjan MT, NGugen T, Srivastava S, Sharma $S$ et al. Frequency of aberrant subclavian artery, arch laterality, and associated intracardiac anomalies detected by echocardiography. Am J Cardiol 2008; 101: 677-682.

8. Felson B, Cohen S, Courter S, McGuire J. Anomalous right subclavian artery. Radiology 1950; 54: 340-349.
9. Patiniotis TC, Mohajeri M, Hill DG. Right aortic arch with aberrant left subclavian artery: aneurysmal dilatation causing symptomatic compression of the right main bronchus in an adult. Aust N Z J Surg 1995; 65: 690-692.

10. Reece TB, Wallen TJ, Jagasia DH, Bavaria JE, SzETo WY. Open thoracic aortic repair for dysphagia in patients with Kommerell's diverticulum and right-sided aortic arch with aberrant left subclavian artery. J Card Surg 2010; 25: 62-64.

11. Myers PO, Fasel JH, Kalangos A, Gailloud P. Arteria lusoria: Developmental anatomy, clinical, radiological and surgical aspects. Ann Cardiol Angeiol 2010; 59: 147-154. 